\title{
La inversión en publicidad y su efecto en las Medianas Empresas de Guayaquil
}

\section{The Investment in advertising and its effect on the Medium Enterprises of Guayaquil}

\begin{abstract}
Catalina Falquez Arce es profesora/investigadora de la carrera de ingeniería en marketing y negociación comercial de la Universidad de Guayaquil (Ecuador) (catalina.falqueza@ug.edu.ec) (http://orcid.org/0000-00030954-3290)
\end{abstract}

Blanca Silva Guerrero es profesora/investigadora de la carrera de ingeniería en marketing y negociación comercial de la Universidad de Guayaquil (Ecuador) (blanca.silvag@ug.edu.ec) (http://orcid.org/orcid.org/00000003-4140-4856)

Vanessa Rojas Herrera es funcionaria de auditoría tributaria en el Servicio de Rentas Internas (Ecuador) (vcrojas@sri.gob.ec) (http://orcid.org/0000-0002-0654-1110)

\begin{abstract}
Resumen
El análisis de las alternativas para mejorar las ventas en las medianas empresas puede presentar el dilema sobre qué tan efectivo será invertir en publicidad. Si bien es cierto que referentes de la aplicación de estrategias de mercadotecnia confirman los efectos de información, persuasión y recordación que obtiene la publicidad, los actuales contextos económicos y sociales exigen mejores argumentos de apoyo. Por lo tanto, y a pesar de que pueden presentarse un sinnúmero de variables relacionadas a otros componentes del marketing, de gestión administrativa, o financiera; el presente estudio procuró determinar el efecto de la publicidad con relación a las ventas de las medianas empresas de Guayaquil en el año 2015, aplicando el modelo de regresión lineal. Como principales resultados se logra determinar que la elasticidad de la variable dependiente «In Ventas» respecto de la variable independiente «In Gastos» de Publicidad es de 3.21, concluyendo que las ventas son influenciadas por los cambios en los gastos de publicidad, corroborando las afirmaciones establecidas desde los diferentes referentes teóricos sobre la importancia de una campaña publicitaria efectiva y la necesidad de empezar a medirla como inversión y no como un gasto.
\end{abstract}

\begin{abstract}
The Analysis of alternatives to improve sales in medium-sized companies may present the dilemma about how effective it will be to invest in Advertising; Although referents of the application of marketing strategies confirm the effects of information, persuasion and recall obtained by advertising, the current economic and social contexts demand better arguments of support. Therefore, and despite the fact that there may be a number of variables related to other marketing, administrative, or financial components; the study sought to determine the effect of advertising in relation to sales of midsize companies in Guayaquil in 2015, using the linear regression model, achieving determining the elasticity of the In dependent variable sales over the independent variable In Advertising Expenses is 3.21, concluding that sales are influenced by changes in advertising expenses, corroborating the affirmations established in the different theoretical references, the importance of an effective advertising campaign and the need to start measuring it as an investment And not as an expense.
\end{abstract}

\section{Palabras clave | keywords}

Mercadotecnia, empresas, ventas, gastos, publicidad, inversión. Marketing, business, sales, advertising, expenses, investment. 


\section{Introducción}

Uno de los retos de los empresarios es alcanzar resultados sostenibles en el tiempo que le permitan mantener el principio del «negocio en marcha». Algunos estudios al respecto afirman que para que esto suceda la empresa debe estar orientada al mercado logrando ventaja competitiva, enfatizando en la generación continua de valor para el comprador y tratando de asegurar su supervivencia a mediano y largo plazo (Álvarez, Santos y Vázquez, 2001; Kohli y Jaworski, 1990; Narver y Slater, 1990). Muchas veces se encuentran Pequeñas y Medianas Empresas (PYMES) que por desconocimiento o falta de presupuesto aún están atrapadas en los enfoques pasivos, es decir enfocadas en producir o comercializar productos y que al tratar de venderlos no tienen clientes fieles, pues los productos o servicios no satisfacen sus expectativas. Los inversionistas de estas compañías tienen que realizar un cambio de enfoque, involucrando un marketing activo en sus estrategias. Analizando algunos postulados sobre el marketing se puede llegar a la conclusión de que es evidente que las organizaciones deben prestar atención a los mecanismos necesarios para captar valor desde y para sus clientes, refrendándose el Marketing como un intercambio de valor entre individuos y organizaciones para poder alcanzar lo que necesitan y así satisfacer las necesidades (Armstrong \& Kotler, 2013). Es interesante observar cómo en las nuevas orientaciones ya se empieza a destacar en las empresas la importancia de crear y mantener las relaciones con los clientes, pues no solo se debe delimitar a la aplicación de un «plan de marketing», sino que se deben saber gestionar las relaciones de intercambio con sus clientes luego de haberse aplicado las estrategias de Marketing Mix.

Afirma Rojas (2016) que además en las nuevas corrientes se da importancia al auge de la tecnología y cómo la empresa debe afrontar la nueva forma de captar clientes y sobrevivir a la economía colaborativa y digital. Por su parte Kotler et al. (2002, pp. 48-51) incluye los últimos conceptos de Marketing definidos por la AMA (2004) como un conjunto de procesos que identifican, crean y distribuyen valor para desarrollar relaciones satisfactorias y de largo plazo que coadyuven a alcanzar beneficios empresariales.

Por lo tanto, no solamente los empresarios, sino los especialistas en marketing, deben reconocer la importancia de la toma de decisiones financieras, creando un contexto de seguridad que apoyará la aplicación de estrategias de marketing de éxito. (Dominguez Doncel \& Muñoz Vera, 2010). 
Monferrer (2013) afirma que es significativo que la comunicación actúe sobre los clientes actuales y potenciales; de allí la importancia de la publicidad como mecanismo para dar a conocer estos productos a los consumidores que están demandando. Según Rojas (2016) citando a Talaya et al. (2008), la publicidad se define como un proceso de comunicación que es realizado por el emisor (vendedor) hacia un grupo de receptores (consumidores reales o potenciales), con el objetivo de dar a conocer los beneficios de valor de sus productos o servicios, persuadiéndolos para su compra.

Para Kotler (2001) una de las más complejas decisiones dentro del marketing es la fijación del presupuesto de promoción y publicidad debido a que deben ser efectivos al momento de asignar presupuesto a la promoción de ventas, publicidad, relaciones públicas, mercadeo directo y fuerza de ventas, en la medida que lo necesita. Existen algunas formas que las empresas utilizan para asignar recursos a este rubro, siendo una de las más utilizadas la fijación de metas en base al porcentaje de venta. Sin embargo, para el autor (op. cit), el método más beneficioso es el de «objetivo y tarea», ya que necesita que se fijen primero los objetivos de la publicidad y luego se definan los costos de cada actividad que se va a requerir.

Es importante también tomar en consideración la estrategia que se va a proponer para lograr alcanzar los objetivos definidos en los presupuestos de publicidad. En este sentido Kotler (2001) detalla cuatro factores que deberían tomarse en consideración al momento de hacer el presupuesto de publicidad: i) el ciclo de vida del producto; ii) la participación en el mercado; iii) la competencia y; iv) la posibilidad de sustitución del producto.

De acuerdo con Rojas (2016), los presupuestos de promoción y publicidad pueden resultar onerosos para las compañías, en especial para las Pequeñas y Medianas (PYMES). Debido a esto, en el momento de la toma de decisiones respecto a la inversión en recursos que generen ingresos, es precisamente la publicidad uno de los rubros que origina ciertas dudas. Por esta razón es común que se dé como práctica relevar los gastos destinados a comunicación y marketing a aspectos como talento humano o producción, ya que no está claramente determinada la influencia o no de la publicidad en los resultados de la empresa.

En el presente trabajo de investigación no se proyectó evaluar cómo las medianas empresas establecieron los presupuestos de promo- 
ción y publicidad, o si los métodos utilizados fueron los idóneos, sino que tiene por objeto analizar cómo los gastos de promoción y publicidad reflejados en sus estados de resultados causaron un efecto en el incremento de sus ventas. Sin embargo, considerando que todo empresario busca maximizar las utilidades de la empresa, es importante conocer si sus recursos están siendo invertidos eficientemente. Por lo tanto, se intenta valorar cuál es el efecto de la inversión de la publicidad en los resultados de las ventas, tomándose como marco muestral las medianas empresas de Guayaquil (Guayas, Ecuador) durante el año 2015.

\section{Metodología}

Según Hernández-Sampieri (2006), la recopilación de información es la forma de recolección de datos sobre variables propias de lo que se analiza. La presente investigación utiliza un enfoque cuantitativo, debido a que, como lo mencionan los autores referidos anteriormente, se necesita recoger datos para poder probar con un análisis estadístico, en este caso puntual, el efecto que tiene la inversión de publicidad en las ventas de las medianas empresas de Guayaquil. Se utilizó el método deductivo, en razón de que se inicia examinando estudios, teorías, análisis y escritos realizados a nivel mundial sobre el impacto de la inversión en publicidad en los rendimientos de las empresas. El tipo de investigación es causal, mientras que el diseño es cuantitativo.

\subsection{Población y Muestra}

El sujeto de estudio son las medianas empresas de Guayaquil. Para llegar a la muestra se siguieron varios criterios de descarte, analizando la base de empresas publicada por la Superintendencia de Compañías del Ecuador. Se parte de la definición de Pequeñas y Medianas empresas tipificadas en el Código Orgánico de la Producción, Comercio e Inversiones. Según el reporte de la Superintendencia de Compañías, se establece que las empresas están clasificadas por su tamaño en Micro, Pequeñas, Medianas y Grandes. Con la finalidad de conseguir una base de datos selecta, se utilizó información del Servicio de Rentas Internas. Según los datos correspondientes a esta base, el universo queda establecido en 46.893 empresas activas y gestionables, registradas en el Registro Único de Contribuyentes de la Zonal 8 (constituida por los cantones de Guayaquil, Samborondón y Durán). Partiendo de este grupo de datos, con la finalidad de constituir 
la muestra de las medianas empresas de la ciudad de Guayaquil, se llevó a cabo el descarte de 6.545 empresas que se hallaban supervisadas por otros organismos diferentes a la Superintendencia de Compañías.

Una vez ejecutado el primer descarte, quedaron 40348 empresas controladas por la Superintendencia de Compañías; de ellas se descartaron 3280 que pertenecían a los cantones de Samborondón y Daule, habiendo, por diferenciación, 37068 empresas de la ciudad de Guayaquil. Posteriormente fueron ordenados los datos por ramas o sectores de la economía basados en el código CIIU1․ En base a este juicio, se exceptúan de la base de datos 6283 empresas que incumben a 12 sectores de actividades de escasa representación comercial y que pueden distorsionar los resultados del análisis, por lo tanto, de esta tercera criba, quedaron configuradas 30785 empresas.

A continuación, y bajo el criterio de empresas que presentaron su declaración de Impuesto a la Renta del año 2015, se seleccionaron 20 438 , pues se entiende que son empresas con actividad comercial actual. De ese grupo se marginaron las empresas cuyos ingresos totales expresados en la declaración de Impuesto a la Renta fluctuaron entre USD 1 000 001,00 hasta USD 5000 000,00, que corresponde a la clasificación de medianas empresas según el Código Orgánico ut supra reseñado. Como consecuencia de esta cuarta criba se obtuvieron 2174 medianas empresas de la ciudad de Guayaquil, las cuales corresponden a la muestra efectiva para el presente estudio y que se presentan por rubro, ventas e inversión publicitaria en la tabla 1.

\subsection{Hipótesis}

Esta investigación intenta como hipótesis de partida determinar si al asignar capital para publicidad se incrementarían las ventas de las empresas en análisis. Una segunda hipótesis plantea comprobar si las ventas se aumentan además por la influencia de la cuota de mercado.

\subsection{Variables}

El estudio realiza el análisis de tres variables: i) monto de ventas (variable dependiente); ii) total de gasto de promoción y publicidad (variable

1 Clasificación sistemática de todas las actividades económicas cuya finalidad es la de establecer su codificación armonizada a nivel mundial. 
independiente) y; iii) cuota de mercado (variable independiente). Esta última se consideró debido a que las ventas determinan la participación de mercado. Esta información corresponde a datos de rubros contables expresados en USD, cuya información fue adquirida de fuentes secundarias como la base de datos de la Superintendencia de Compañías y del Servicio de Rentas Internas, según se observa en las declaraciones reportadas por las empresas como resultados del ejercicio económico 2015. Cabe indicar que para el estudio no se consideran otras variables que también pueden incidir en los resultados de la empresa, como descuentos en ventas y otras promociones y estrategias de venta personal o de distribución, debido a que el tema central del análisis es la publicidad y su importancia como herramienta de comunicación, persuasión y memorización del mensaje de venta.

Tabla 1. Extracto de $n$ de Medianas Empresas de Guayaquil por Sector

\begin{tabular}{|l|l|l|l|}
\hline \multicolumn{1}{|c|}{ SECTOR } & NORDEN & $\begin{array}{r}\text { TOTAL } \\
\text { DE VENTAS }\end{array}$ & $\begin{array}{c}\text { TOTAL DE } \\
\text { GASTO DE } \\
\text { PUBLICIDAD }\end{array}$ \\
\hline $\begin{array}{l}\text { Actividades de alojamiento y de servi- } \\
\text { cio de comidas. }\end{array}$ & 49 & 98518570,35 & 837028,60 \\
\hline $\begin{array}{l}\text { Actividades de atención de la salud } \\
\text { humana y de asistencia social. }\end{array}$ & 59 & 128739490,20 & 389772,18 \\
\hline Actividades inmobiliarias. & 73 & 128236236,21 & 1137099,18 \\
\hline $\begin{array}{l}\text { Actividades profesionales, científicas } \\
\text { y técnicas. }\end{array}$ & 159 & 321740118,79 & $6.108297,47$ \\
\hline $\begin{array}{l}\text { Agricultura, ganadería, silvicultura y } \\
\text { pesca. }\end{array}$ & 261 & 526949003,31 & 250256,33 \\
\hline $\begin{array}{l}\text { Comercio al por mayor y al por menor; } \\
\text { reparación de vehículos automotores } \\
\text { y motocicletas. }\end{array}$ & 976 & 2160955593,12 & 11661466,14 \\
\hline Construcción. & 162 & 321893390,89 & 292472,80 \\
\hline Industrias manufactureras. & 258 & 572782166,62 & 3410424,45 \\
\hline Otras actividades de servicios. & 32 & 54780740,13 & 281633,86 \\
\hline Transporte y almacenamiento. & 145 & 294650992,24 & 484525,29 \\
\hline Total USD (US\$) & 2174 & 4609246301,86 & 24852976,30 \\
\hline
\end{tabular}

Fuente: Servicio de Rentas Internas

Nota: *Tomado de Rojas (2016) 


\subsection{Análisis estadístico}

Según Gujarati \& Porter (2010), existen modelos que pueden ser lineales en los parámetros. Sin embargo en las variables no lineales requieren una transformación de las variables para obtener una mejor interpretación de los resultados del modelo de regresión. Así, el presente estudio utiliza el modelo Log-lineal, y como herramienta fue utilizado XLSTAT, con el cual se aplicó este modelo de regresión lineal.

Para aplicar las fórmulas de regresión lineal, en el caso de la variable dependiente, se tomaron datos de los ingresos publicados por el servicio de rentas internas que se relacionan con sus movimientos habituales como son las ventas, monto que asciende a USD 4609246301,86 en el total ventas declaradas (ver tabla 1 y 2). En el caso de la variable independiente «gastos de publicidad», se eligieron los montos declarados en los servicios tributarios bajo el concepto «costos y gastos de promoción y publicidad», cantidades que se incorporaron en relación del presente análisis: USD 24852 976,30 (ver tabla 1).

Para Gujarati \& Porter (2010), emplear exclusivamente dos variables, la dependiente y la explicativa, no es tan real en la práctica. La situación es que sobre la variable «ventas» pueden existir muchas variables que la pueden afectar; por lo tanto, en la presente investigación se incluye otra variable independiente como es la «cuota de mercado». Para determinar el valor correspondiente a esta variable se utilizó la información de la variable «ventas», explicada en párrafos anteriores; estableciéndose así el valor total de ventas operacionales de cada sector económico. Posteriormente se obtuvo el porcentaje de las ventas de cada mediana empresa de Guayaquil, lográndose determinar de esta manera la cuota de mercado correspondiente a su sector, como se observa en la tabla 2.

Tabla 2. Declaración de Impuesto a la Renta Año 2015 2175 Medianas Empresas de Guayaquil

\begin{tabular}{|l|l|l|}
\hline \multicolumn{1}{|c|}{ CAS. } & \multicolumn{1}{|c|}{ DESCRIPCIÓN } & \multicolumn{1}{c|}{ VALOR USD } \\
\hline 6999 & Total, de ingresos declarados & $4.687 .592 .567,54$ \\
\hline Casilleros escogidos correspondiente a ingresos operacionales: \\
\hline 6001 & $\begin{array}{l}\text { Ventas netas locales gravadas con tarifa 12\% } \\
\text { de IVA }\end{array}$ & $2.380 .221 .449,47$ \\
\hline 6005 & $\begin{array}{l}\text { Prestaciones locales de servicios gravadas } \\
\text { con tarifa 12\% de IVA }\end{array}$ & $655.389 .176,26$ \\
\hline
\end{tabular}




\begin{tabular}{|c|c|c|}
\hline CAS. & DESCRIPCIÓN & VALOR USD \\
\hline 6002 & $\begin{array}{l}\text { Valor exento ventas netas locales gravadas } \\
\text { con tarifa } 12 \% \text { de IVA }\end{array}$ & $49.193,47$ \\
\hline 6003 & $\begin{array}{l}\text { Ventas netas locales gravadas con tarifa cero } \\
\text { o exentas de IVA }\end{array}$ & $976.711 .407,17$ \\
\hline 6007 & $\begin{array}{l}\text { Prestaciones locales de servicios gravadas } \\
\text { con tarifa cero o exentas de IVA }\end{array}$ & $247.224 .527,20$ \\
\hline 6008 & $\begin{array}{l}\text { Valor exento prestaciones locales de servicios } \\
\text { gravadas con tarifa cero o exentas de IVA }\end{array}$ & $1.166 .750,33$ \\
\hline 6004 & $\begin{array}{l}\text { Valor exento ventas netas locales gravadas } \\
\text { con tarifa cero o exentas de IVA }\end{array}$ & $2.968 .933,48$ \\
\hline 6009 & Exportaciones netas de bienes & $161.366 .315,62$ \\
\hline 6011 & Exportaciones netas de servicios & $47.046 .720,67$ \\
\hline 6012 & Valor exento exportaciones netas de servicios & $1.344 .995,19$ \\
\hline 6010 & Valor exento exportaciones netas de bienes & $11.833,68$ \\
\hline 6013 & $\begin{array}{l}\text { Ingresos por prestación de servicios de cons- } \\
\text { trucción }\end{array}$ & $103.532 .370,96$ \\
\hline 6091 & Otros ingresos del exterior & $2.204 .075,67$ \\
\hline 6015 & $\begin{array}{l}\text { Ingresos obtenidos bajo la modalidad de } \\
\text { comisiones o similares }\end{array}$ & $13.890 .740,35$ \\
\hline 6092 & Valor exento otros ingresos del exterior & $1.289 .397,29$ \\
\hline 6017 & $\begin{array}{l}\text { Ingresos obtenidos por arrendamientos } \\
\text { operativos }\end{array}$ & $14.828 .415,05$ \\
\hline \multicolumn{2}{|c|}{ Total de ventas base para el análisis } & 4.609.246.301,86 \\
\hline
\end{tabular}

Fuente: Servicio de Rentas Internas

\subsection{Modelo Log-lineal}

En razón de que los datos de las variables «ventas», «gasto de publicidad» y «cuota de mercado», están muy disgregados; se transformaron las variables a «ln», lo cual va a expresar mejor el modelo. Una vez explicadas las variables que se utilizan en el modelo de regresión lineal múltiple, se procura señalar las relaciones de dichas variables y el efecto de la inversión en publicidad sobre el horizonte de las ventas de las medianas empresas de Guayaquil, en términos porcentuales.

\section{Resultados}

Al integrarse los datos referidos ut supra en la herramienta estadística XLSTAT, se encuentra que la variable explicativa es el gasto de promoción 
y publicidad expresado en «ln», y la variable respuesta es el «ln Ingresos por ventas»; es decir que la variable de «promoción y publicidad» puede manifestar el nivel de ventas de las medianas empresas de Guayaquil. Los datos presentan una relación lineal directamente proporcional.

\subsection{Estadísticos descriptivos}

Con un intervalo de confianza del 95\% y un nivel de tolerancia del 0.0001, a continuación se presentan los estadísticos descriptivos de las variables estudiadas:

Tabla 3. Estadísticos descriptivos de las variables analizadas

\begin{tabular}{|l|l|l|l|l|l|l|l|}
\hline \multicolumn{1}{|c|}{ Variable } & $\begin{array}{c}\text { Observa- } \\
\text { ciones }\end{array}$ & $\begin{array}{c}\text { Obs. con } \\
\text { datos } \\
\text { perdidos }\end{array}$ & $\begin{array}{c}\text { Obs. sin } \\
\text { datos } \\
\text { perdidos }\end{array}$ & Mínimo & Máximo & Media & $\begin{array}{c}\text { Desv. } \\
\text { típica }\end{array}$ \\
\hline In Ventas & 1093 & 0 & 1093 & 13,545 & 15,419 & 14,527 & 0,46 \\
\hline $\begin{array}{l}\text { In Gastos } \\
\text { de Publicidad }\end{array}$ & 1093 & 0 & 1093 & 1,902 & 14,66 & 8,111 & 2,143 \\
\hline $\begin{array}{l}\text { In cuota } \\
\text { de mercado }\end{array}$ & 1093 & 0 & 1093 & $-7,806$ & $-2,686$ & $-5,997$ & 1,131 \\
\hline
\end{tabular}

Los estadísticos descriptivos de la variable «ln ventas» revelan una desviación típica de 0.46, con una media de 14,527 en un rango entre 12,545 y 15,419 , mientras en el caso de la variable «ln gasto de publicidad» se presenta una desviación típica de 2,143. Además se pretende determinar si el coeficiente $\mathrm{R}^{2}$ se ajusta al perfil de regresión lineal a los datos. Así, en el modelo se establece el grado de relación de las variables, no una relación de causalidad.

Gujarati \& Porter (2010) mencionan que existen muchos investigadores tratando de ajustar sus modelos a un coeficiente cercano a 1 para demostrar la efectividad de los mismos. Sin embargo, los precitados autores mencionan que el investigador debe centrarse más en los resultados estadísticos de las variables explicativas con respecto a la variable dependiente. De esta forma concluyen que el hecho de que el resultado del coeficiente de determinación esté alejado del 1, no significa que el modelo sea malo. En la tabla 4 se presentan los resultados de regresión de la variable «In ventas» con bondad del ajuste, emergiendo que el coeficiente de determinación $\mathrm{R}^{2}$ es de $12 \%$. 
Tabla 4. Regresión Lineal de las Variables

\begin{tabular}{|l|l|}
\hline \multicolumn{2}{|c|}{$\begin{array}{c}\text { Regresión de la variable In VENTAS } \\
\text { Estadísticos de bondad del ajuste (In vtas) }\end{array}$} \\
\hline Observaciones & 1093 \\
\hline Suma de los pesos & 1093 \\
\hline GL & 1090 \\
\hline$R^{2}$ & 0,124 \\
\hline$R^{2}$ ajustado & 0,122 \\
\hline
\end{tabular}

\subsection{Análisis de Varianza}

Se logra determinar la variabilidad de «In ventas» por medio de la información obtenida del análisis de varianza de las dos variables independientes (tabla 5).

Tabla 5. Análisis de Varianza y de suma de cuadrados

\begin{tabular}{|c|c|c|c|c|c|}
\hline \multicolumn{6}{|c|}{ Análisis de varianza (In ventas) } \\
\hline Fuente & $\mathrm{L}$ & $\begin{array}{c}\text { Suma de } \\
\text { cuadrados }\end{array}$ & $\begin{array}{c}\text { Cuadrados } \\
\text { medios }\end{array}$ & & $r>F$ \\
\hline Modelo & & 8,609 & 4,305 & 7,024 & 0,0001 \\
\hline Error & 090 & 02,429 &, 186 & & \\
\hline Total corregido & 092 & 31,039 & & & \\
\hline \multicolumn{6}{|c|}{ Calculado contra el modelo $Y=$ Media(Y) } \\
\hline \multicolumn{6}{|c|}{ Análisis Suma de Cuadrados Tipo I (In vtas) } \\
\hline Fuente & $\mathrm{L}$ & $\begin{array}{c}\text { Suma de } \\
\text { cuadrados }\end{array}$ & $\begin{array}{c}\text { Cuadrados } \\
\text { medios }\end{array}$ & & $r>F$ \\
\hline In Gasto de publicidad & & ,888 & ,888 & 1,706 & 0,0001 \\
\hline In Cuota de mercado & & 2,721 & 2,721 & 22,343 & 0,0001 \\
\hline \multicolumn{6}{|c|}{ Análisis Suma de Cuadrados Tipo III (In vtas) } \\
\hline Fuente & $\mathrm{L}$ & $\begin{array}{c}\text { Suma de } \\
\text { cuadrados }\end{array}$ & $\begin{array}{c}\text { Cuadrados } \\
\text { medios }\end{array}$ & & $r>F$ \\
\hline In Gasto de publicidad & &, 17 &, 17 & 7,841 & 0,0001 \\
\hline In Cuota de mercado & & 2,721 & 2,721 & 22,343 & 0,0001 \\
\hline
\end{tabular}

Debido a que el valor $\mathrm{p}$ (valor de probabilidad) es mayor que el estadístico $\mathrm{F}$ en el modelo y por ende en las dos variables independientes, se puede interpretar que la información dada por las variables independientes «ln gastos de publicidad» e «ln cuota de mercado» explica 
significativamente la variabilidad de «ln ventas», así como la influencia de la variable «cuota de mercado».

\subsection{Análisis de la regresión}

Finalmente se determina la elasticidad de la variable dependiente «In ventas» respecto de la variable independiente «In gastos de publicidad».

Tabla 6. Análisis de la Regresión

\begin{tabular}{|l|l|l|l|l|l|l|}
\hline \multicolumn{7}{|c|}{ Parámetros del modelo (Ln vtas) } \\
\hline Fuente & Valor & $\begin{array}{l}\text { Error } \\
\text { estándar }\end{array}$ & $T$ & Pr>|t| & $\begin{array}{l}\text { Límite } \\
\text { inferior } \\
(95 \%)\end{array}$ & $\begin{array}{l}\text { Límite } \\
\text { superior } \\
(95 \%)\end{array}$ \\
\hline Intercepción & 15,032 & 0,087 & 172,269 & $<0,0001$ & 14,861 & 15,203 \\
\hline $\begin{array}{l}\text { In Gasto de } \\
\text { publicidad }\end{array}$ & 0,032 & 0,006 & 5,276 & $<0,0001$ & 0,02 & 0,044 \\
\hline $\begin{array}{l}\text { In Cuota de } \\
\text { Mercado }\end{array}$ & 0,128 & 0,012 & 11,061 & $<0,0001$ & 0,105 & 0,15 \\
\hline
\end{tabular}

Ecuación del modelo Regresión Lineal (In Ventas):

Como resultado de la aplicación, la elasticidad de la variable dependiente «ln ventas» respecto de la variable independiente «ln gastos de publicidad» es de 3.21, lo que indica que si las otras variables permanecen constates, al aumentarle $1 \%$ en «ln gasto de publicidad», le corresponde un aumento de $3.21 \%$ en «ln ventas», por lo que se evidencia con meridiana claridad que en el caso de análisis las ventas son impactadas directamente por los cambios en los gastos de publicidad.

\section{Discusión}

Los resultados de la presente investigación demuestran la importancia que se está dando a la variable de inversión en promoción y publicidad, con respecto a la variación en ventas en un grupo de Medianas Empresas de la ciudad de Guayaquil. Es significativo aclarar que la inversión en promoción y publicidad reflejada como un gasto en el estado de resultados de una compañía no es la única variable que afecta a la variación en ventas, como ya se mencionó en referencia. 
Sin embargo el estudio es coherente con las manifestaciones de otros autores que demuestran que las ventas y los beneficios están directamente relacionados con la publicidad y que son variables importantes para poder evaluar si realmente se está siendo efectivo en la inversión de publicidad, siendo el beneficio el producto del margen unitario por las unidades vendidas. No obstante, para todas las empresas no emerge el mismo resultado debido a que puede variar por diferentes motivos como costos diferentes, precios, entre otros (Tellis \& Redondo, 2002).

Kotler (2001) afirma la importancia de la mercadotecnia y sus estrategias -entre las que se encuentra la publicidad-, ya que a medida que la empresa empieza a darle más fuerza, su potencial de ventas se va acercando a su mercado potencial, estableciendo una relación directa entre el nivel de ventas y el nivel de mercadotecnia, lo cual se evidencia en el análisis realizado.

Cabe mencionar otros estudios con resultados similares al presente, considerando el estudio efectuado por Sellamen-Garzón \& ArceMeza, (2010) sobre las decisiones publicitarias y su influencia en las ventas de los productos en tiendas de Bogotá, quienes concluyen que aplicando el método de regresión lineal se evidencia una relación directa entre las cantidades demandadas y la inversión en publicidad.

Tellis \& Redondo (2002) por su parte señalan que existen algunas investigaciones sobre la relación publicidad-ventas, las cuales han aportado buenos resultados sobre los efectos de la publicidad, así como ciertos estudios de campo que han alcanzado obtener como resultado que si se aumenta la presión publicitaria en el $1 \%$, se va a obtener un incremento del $0.01 \%$ en las ventas.

\section{Conclusión}

Según el modelo de regresión lineal múltiple aplicado en el presente trabajo de investigación con los datos de las medianas empresas de la ciudad de Guayaquil, se concluye que las variables: a) ventas, b) gasto de publicidad y c) cuota de mercado, están directamente relacionadas, siendo la variable «ventas» dependiente de las variables explicativas «gasto de publicidad»y «cuota de mercado».

Asimismo se demostró que a medida que se incrementa el gasto de publicidad crece el nivel de ventas, resultados que corroboran las conclusiones establecidas en los diferentes referentes teóricos mencio- 
nados en el presente trabajo de investigación. Debido a que muchos de los autores citados ut supra estudian al Marketing desde una perspectiva teórica, destacan la importancia de la aplicación de estrategias de mercadotecnia con el objetivo de satisfacer al consumidor, crear relaciones de intercambio de valor y por ende alcanzar las metas de las empresas, maximizando las utilidades de los accionistas o inversores.

Se debe mencionar que en los resultados de la investigación se pudo evidenciar que las medianas empresas de la ciudad de Guayaquil no están destinando los suficientes recursos al rubro de marketing, lo que se observa en los valores señalados como gasto de publicidad en las declaraciones de impuesto a la renta, en los que esta partida presupuestaria declarada representa -en promedio- un $0.54 \%$ con respecto a los ingresos operacionales; aspecto que requiere un análisis en posteriores investigaciones.

La presente investigación no analiza la causa de los niveles de gasto en publicidad de cada sector económico de las medianas empresas, por ello es recomendable realizar a futuro un estudio a medianas empresas de sectores explícitos de las diferentes regiones del país, con el fin de analizar su variación de inversión en publicidad y si ésta tiene efecto en las ventas, incluso pudiéndose diseñar un patrón más completo con otras variables independientes. Además, con las actuales reformas tributarias en el Ecuador sobre los procesos de deducción del gasto de publicidad, otro estudio a futuro podría ser el análisis del impacto de dicha deducción en los procesos de toma de decisiones de las medianas y grandes empresas en esta materia.

\section{Referencias}

AMA (2013). Definición de Marketing. Recuperado el 16 de Jullio de 2016, de https:// www.ama.org

Armstrong, G., \& Kotler, P. (2013). Fundamentos del Marketing. México: Pearson Educación de México.

Clow, K. E., \& Baack, D. (2010). Publicidad, Promoción y Comunicación Integral en Marketing ( $4^{\underline{a}}$ Ed). México: Pearson Educación.

Dominguez-Doncel, A., \& Muñoz-Vera, G. (2010). Métricas del Marketing (2 ${ }^{\underline{a}}$ Ed). Madrid: Esic Editorial.

Garza-Castaño, R. (2000). Creación de PYMES: Objetivo emprendedor. Ingenierías, 54-58.

Gujarati, D. N., \& Porter, D. (2010). Econometría (5ํㅗ Ed). México: McGraw-Hill/ Interamericana Editores.

Hernández-Sampieri, Fernández \& Baptista (2006). Metodología de la Investigación (4⿻ Ed). México: Pearson Education. 
Kelmansky, D. M. (2009). Estadística para Todos. Buenos Aires: Ministerio de Educación-Instituto Nacional de Educación Tecnológica.

Kotler, P. (2001). Dirección de Mercadotecnia, Análisis, Planeación, Implementación y Control ( $8^{\underline{a}}$. Ed). Lima: Pearson Educación.

Monferrer, D. (2013). Fundamentos de Marketing. Castellón de la Plana: Unión de Editoriales Universitarias Españolas.

Rojas, V. (2016). Efecto financiero de la inversión de Publicidad en los resultados de las Medianas empresas de Guayaquil.

Sellamen-Garzón, A., \& Arce-Meza, A. (2010). Las decisiones publicitarias dependen de las ventas en los Productos. Criterio Libre, 8(12), 143-161.

Talaya, A. E., García-De-Madariaga-Miranda, J., Narros-González, M. J., Olarte.Pascual, C., Reinares-Lara, E. M., \& Saco-Vázquez, M. (2008). Principios de Marketing $\left(3^{\underline{a}} \mathrm{Ed}\right)$. Madrid: Esic Editorial.

Tellis, G. J., \& Redondo, I. (2002). Estrategias de Promoción y Publicidad. Madrid: Pearson Education. 